\title{
Transtorno de Déficit de Atenção e Hiperatividade em crianças: análise de artigos nacionais da última década (2003-2013)
}

\author{
Miriam Maria Meque*, Zaida Aurora Sperli Geraldes Soler, D.Sc.**
}

*Acadêmica do Curso de Graduação em Enfermagem da Faculdade de Medicina de São José do Rio Preto - FAMERP,

**Obstetriz, Enfermeira, Docente em Enfermagem Obstétrica, Docente e Orientadora da Graduaçâo e da Pós-graduação da FAMERP, Coordenadora do Programa de Enfermagem - Mestrado Acadêmico da FAMERP

\begin{abstract}
Resumo
Introdução: O Transtorno de Déficit de Atenção e Hiperatividade (TDAH) é um problema de grande prevalência na infância e adolescência. Caracteriza-se por distúrbios motores, perceptivos, emocionais e comportamentais. Objetivo: $\mathrm{O}$ objetivo deste estudo foi analisar artigos científicos de autores brasileiros na última década. Método: Revisão bibliográfica de artigos nacionais indexados na Biblioteca Virtual de Saúde (BVS) da rede Bireme. Resultados: Foram resenhados 185 artigos, veiculados online, referentes ao período de 2003 a 2013. Conclusão: O TDAH merece estudos mais amplos e a partir dos primeiros anos de vida, de modo a entender os sinais e sintomas e as várias comorbidades envolvidas.
\end{abstract}

Palavras-chave: transtorno de deficit de atenção, hiperatividade, crianças, transtorno do comportamento infantil.

\begin{abstract}
Attention Deficit Disorder with Hyperactivity among children: an analysis of national articles published within the last decade (2003-2013)

Introduction: Attention Deficit Disorder with Hyperactivity (ADDH) is a problem with high prevalence rates in childhood and adolescence. It is characterized by motor, perceptual, emotional and behavioral disorders. Objective: The objective of this study was to analyze scientific articles from Brazilian authors published within the last decade. Method: Bibliographic review of national articles indexed on Bireme's Virtual Health Library. Results: 185 articles available online and published from 2003 to 2013 were reviewed. Conclusion: Further research on ADDH (especially in the early years of a child's life) is necessary in order to understand its signs, symptoms and related comorbidities.
\end{abstract}

Key-words: attention deficit disorder, hyperactivity, child, child behavior disorders. 


\section{Resumen}

\section{Trastorno por Déficit de Atención e Hiperactividad entre niños: análisis de artículos nacionales publicados en la última década (2003-2013)}

Introducción: El Trastorno por Déficit de Atención e Hiperactividad (TDAH) es un problema de gran prevalencia en la infancia y adolescencia. Se caracteriza por trastornos motores, perceptivos, emocionales y comportamentales. Objetivo: El objetivo de este estudio fue analizar artículos científicos de autores brasileños publicados en la última década. Método: Revisión bibliográfica de artículos nacionales indexados en la Biblioteca Virtual en Salud (BVS) de la red Bireme. Resultados: 185 artículos disponibles en versión electrónica durante el período de 2003-2013 fueron revisados. Conclusión: Son necesarios estudios más amplios sobre el TDAH, sobre todo en los primeros años de vida, para entender mejor sus síntomas, señales y las comorbilidades asociadas.

Palabras-clave: trastorno por déficit de atención, hiperactividad, niños, trastorno de la conducta infantil.

\section{Introdução}

O transtorno do déficit de atenção e hiperatividade (TDAH) é considerado o agravo de desenvolvimento infantil mais diagnosticado na última década, caracterizando-se por padrôes de desatenção e impulsividade/agitação severos e persistentes e comorbidades [1]. Devido à sua alta prevalência, se não for diagnosticado e tratado adequadamente pode ocasionar grandes dificuldades sociais, acadêmicas e familiares, prejudicando a qualidade de vida dos afetados e de seus familiares [1-5]. Os psicólogos e psiquiatras podem contribuir para o desenvolvimento de estratégias mais eficazes de intervenção, pela junção dos saberes e prática da interdisciplinaridade [6,7].

$\mathrm{O}$ diagnóstico de TDAH requer a presença de sintomatologia verificada no âmbito doméstico e na escola, por meio dos relatos de pais e professores, mas ainda é pouco abordado em pesquisas no Brasil [6,7].

O critério diagnóstico do TDAH geralmente é baseado no Manual Diagnóstico e Estatístico das Doenças Mentais [8] e os autores são unânimes em destacar a necessidade de maior interação entre variáveis biológicas e comportamentais na compreensão das bases etiológicas e de tratamento [8-10]. Nos últimos anos tem sido investigada a possibilidade de introdução de diagnóstico psiquiátrico para crianças afetadas com TDAH [11-15].

A inabilidade social das crianças com TDAH é logo percebida pelos pais, cuidadores e professores, que destacam falhas comportamentais como: falta de atenção, agitação, impulsividade, impaciência, nervosismo, rebeldia, além de problemas de aprendizado escolar [9]. Esta população infantil afetada tem grande demanda para os serviços de saúde mental e principalmente nos Estados Unidos são propostos programas de intervençáo educacional, psicológica e medicamentosa [11].

A realização de estudos bibliográficos sobre TDAH, conduzidos de forma sistematizada e científica, é importante, por permitir desvelar as diferentes nuances dessa temática e o panorama das pesquisas no Brasil. Também podem ser reveladas açôes terapêuticas e educacionais de relevância e a contribuição dos diferentes profissionais de saúde na abordagem do TDAH em crianças. Assim, este estudo tem como objetivo: Identificar artigos divulgados de 2003 a 2013 em periódicos científicos nacionais sobre o Transtorno do Déficit de Atenção e Hiperatividade (TDAH) em crianças.

\section{Métodologia}

Trata-se de estudo bibliográfico sistematizado, com uso da Internet e da integração com a Biblioteca Virtual em Saúde (BVS), por meio da Bireme (Centro Latino-Americano e do Caribe de Informação em Ciências da Saúde), nas Bases Eletrônicas Lilacs e SciELO Brasil e pelo endereço eletrônico http://scholar.google.com. br, utilizando-se como descritores: "transtorno"; crianças; hiperatividade.

A pesquisa bibliográfica, seja narrativa, integrativa, sistematizada, envolve tanto descriçôes quantitativas quanto qualitativas, dependendo dos objetivos do estudo e das variáveis que se deseja estudar. Permite o aprofundamento de estudos sobre assunto específico, por meio de referências teóricas com evidências científicas 
publicadas em documentos. As informaçóes bibliográficas científicas são organizadas, classificadas e indexadas em diferentes bancos de dados, com utilização da internet, em redes de informação interligadas a bibliotecas nacionais e internacionais.

Para a coleta dos dados, elaborou-se um instrumento que permitisse extrair os elementos essenciais dos textos e dos periódicos, por meio de elaboraçáo de resenhas. Tomou-se cuidado no sentido de respeitar as ideias explicitadas pelos autores em suas pesquisas. A seleção, classificação e análise das publicaçóes tomaram por base a coerência e pertinência dos documentos para sua seleção e ser divulgado na íntegra, sendo os resultados apresentados em tabelas e na forma descritiva.

\section{Resultados e discussão}

Este estudo constitui-se numa análise artigos científicos de autores brasileiros sobre transtorno do déficit de atenção e hiperatividade (TDAH). Nesta revisão da literatura, encontrou-se 148 artigos científicos de autores brasileiros, publicados entre 2003 e 2013 em periódicos na base de dados da rede Bireme. Os estudiosos sobre o assunto publicaram em 37 periódicos, área de psicologia (63 artigos em 14 periódicos), medicina (55 artigos em 12 periódicos) e Educação ou Saúde Coletiva (30 artigos em 11 periódicos).

A sigla CAPES significa Coordenação de Aperfeiçoamento de Pessoal de Nível Superior e constitui uma agência governamental brasileira, vinculada ao Ministério da Educação e Cultura (MEC), de fomento à pesquisa e expansão e consolidação dos cursos de pós-graduação stricto sensu. A CAPES foi criada em 1951, com a finalidade de formar com qualidade pessoal especializado e foi denominada "Campanha Nacional de Aperfeiçoamento de Pessoal de Nível Superior" [16]. As bases de dados utilizadas como critério de avaliação para o Qualis possuem as seguintes características: Scielo (Scientific Electronic Library Online) pode ser definida como uma biblioteca eletrônica que abrange uma selecionada coleçáo de periódicos científicos latino-americanos em todas as áreas do conhecimento. Foi desenvolvida a partir de uma parceria entre a FAPESP (Fundação de Amparo à Pesquisa do Estado de São Paulo) e a Bireme (Centro Latino-Americano e do Caribe de Informação em Ciências da Saúde), com o apoio do CNPq (Conselho Nacional de Desenvolvimento Científico e Tecnológico). O periódico incluído na coleção Scielo Brasil deve passar por ser avaliado por critérios internacionais das bases de dados e um destes é ser indexado na base Lilacs [17-20].

Medline foi desenvolvida pela National Library of Medicine em 1966 e abrange somente artigos de periódicos científicos internacionais. Os assuntos abrangidos são medicina, enfermagem, odontologia e medicina veterinária $[19,20]$.

Excerpta Medica (Embase) é um banco de dados bibliográfico da área farmacológica e biomédica, mantido pela Elsevier Science e 1.700 periódicos indexados são de publicação exclusiva [20].

Lilacs (Literatura Latino-Americana e do Caribe em Ciências da Saúde) registra a literatura científico-técnica em saúde produzida por autores latino-americanos e do Caribe desde 1982 [20].

A manutenção da base de dados Lilacs e de outras bases temáticas em Ciências da Saúde é de responsabilidade da Bireme, que é um centro especializado da OPAS (Organização Panamericana de Saúde) estabelecido no Brasil desde 1967, em colaboração com a UNIFESP (Universidade Federal de São Paulo). A Bireme é conhecida como Centro Latino-Americano e do Caribe de Informação em Ciências da Saúde. Na Lilacs, livros, teses, capítulos de livros, anais de congressos ou conferências, relatórios técnico-científicos e artigos de revistas relacionados à área da Saúde são descritos e indexados [20].

Anualmente a Capes disponibiliza no sistema Webqualis para consulta a classificação de periódicos, anais, revistas e jornais, em cada área do conhecimento. A classificação de periódicos é realizada pelas áreas de avaliação e passa por processo anual de atualização. Esses veículos são enquadrados em estratos indicativos da qualidade - A1, o mais elevado; A2; B1; B2; B3; B4; B5; C - com peso zero.

Observa-se na Tabela I que os 148 artigos selecionados foram publicados em periódicos das seguintes áreas: Psicologia - 65 (43,91\%); Psiquiatria/ Medicina (55 - 37,16\%) e Saúde Coletiva e outras áreas da saúde 30 (20,27\%). Quanto ao ano de publicação dos artigos verifica-se que foram crescentes a partir de 2003, com maior número nos anos de 2007 ( 28 - 18,91\%), em 2009 (27 - 18,24\%) e em $2011(20-30,51 \%)$. 
Tabela I - Artigos sobre Transtorno de Déficit de Atenção e Hiperatividade (TDAH), segundo ano e área temática dos periódicos.

\begin{tabular}{|c|c|c|c|c|}
\hline \multirow{3}{*}{$\begin{array}{l}\text { Ano } \\
\text { de } \\
\text { Publi- } \\
\text { cação }\end{array}$} & \multicolumn{4}{|c|}{ Área profissional dos periódicos } \\
\hline & Psicologia & $\begin{array}{l}\text { Psiquia- } \\
\text { tria/ } \\
\text { Áreas } \\
\text { Medicina }\end{array}$ & $\begin{array}{l}\text { Educação } \\
\text { Saúde Cole- } \\
\text { tiva/ Outras } \\
\text { áreas }\end{array}$ & TOTAL \\
\hline & $\mathrm{N}^{\circ}(\%)$ & $N^{\circ}(\%)$ & $N^{\circ}(\%)$ & $\mathrm{N}^{\circ}(\%)$ \\
\hline 2003 & $1(1,58)$ & $2(3,63)$ & $1(3,33)$ & $4(2,70)$ \\
\hline 2004 & $1(1,58)$ & $2(3,63)$ & - & $3(2,02)$ \\
\hline 2005 & $3(4,76)$ & $4(6,34)$ & $2(6,66)$ & $8(5,40)$ \\
\hline 2006 & $5(7,93)$ & $4(6,34)$ & $1(3,33)$ & $10(6,75)$ \\
\hline 2007 & $5(7,93)$ & $21(38,18)$ & $2(6,66)$ & $28(18,91)$ \\
\hline 2008 & $13(20,63)$ & $3(5,45)$ & $3(10,00)$ & $19(12,83)$ \\
\hline 2009 & $12(19,04)$ & $8(14,54)$ & $7(23,33)$ & $27(18,24)$ \\
\hline 2010 & $8(12,69)$ & $2(3,63)$ & $3(10,00)$ & $13(6,75)$ \\
\hline 2011 & $11(17,46)$ & $4(6,34)$ & $5(16,66)$ & $20(13,51)$ \\
\hline 2012 & $4(6,34)$ & $3(5,45)$ & $1(3,33)$ & $8(5,40)$ \\
\hline 2013 & $1(1,58)$ & $2(3,63)$ & $5(16,66)$ & $8(5,40)$ \\
\hline TOTAL & 63 (100) & 55 (100) & $30(100)$ & 148 (100) \\
\hline
\end{tabular}

São apresentados a seguir os periódicos da área de Psicologia (Quadro 1), Medicina (Quadro 2) e Educação-Saúde Pública/Outras áreas (Quadro 3), assim como a classificação Qualis Capes 2013 dos periódicos de cada área.

Encontraram-se 63 artigos publicados em 14 periódicos da área de psicologia, $8(57,14 \%)$ tinham qualificação Qualis A, sendo 6 (75\%) A2. Dos 6 periódicos restantes, $4(66,66 \%)$ eram $\mathrm{B} 1,1$ (16,66\%) era B2 e $1(16,66 \%)$ era B3. Já na avaliação pela área de conhecimento Enfermagem, 4 periódicos não tinham qualificação (SQ), 6 eram B1, 2 eram B2, 1 B3 e 1 B4.

Quadro 1 - Denominação dos Periódicos de Psicologia, número de artigos sobre TDAH e Classificação Qualis Capes na área.

\begin{tabular}{|l|c|c|}
\hline \multicolumn{1}{|c|}{ Periódico } & $\begin{array}{c}\text { Número } \\
\text { de artigos }\end{array}$ & $\begin{array}{c}\text { Qualis } \\
\text { Psico- } \\
\text { logia/ } \\
\text { Enferma- } \\
\text { gem }\end{array}$ \\
\hline Psicologia Escolar e Educacional & 12 & $\mathrm{~B} 1 / \mathrm{B} 2$ \\
\hline Psicologia: Reflexão e Crítica & 10 & $\mathrm{~A} 1 / \mathrm{B} 1$ \\
\hline Psicologia: Teoria e Pesquisa & 6 & $\mathrm{~A} 1 / \mathrm{B} 1$ \\
\hline Psicologia USP & 4 & $\mathrm{~A} 2 / \mathrm{B} 1$ \\
\hline Psico-USF & 4 & $\mathrm{~A} 2 / \mathrm{B} 1$ \\
\hline Psicologia em Revista & 4 & $\mathrm{~B} 1 / \mathrm{B} 3$ \\
\hline Fractal: Revista de Psicologia & 3 & $\mathrm{~B} 1 / \mathrm{SQ}$ \\
\hline Boletim de Psicologia & 3 & $\mathrm{~B} 2 / \mathrm{SQ}$ \\
\hline
\end{tabular}

\begin{tabular}{|l|c|c|}
\hline Estudos de Psicologia & 3 & A2/B2 \\
\hline Psicologia \& Sociedade & 3 & A2/SQ \\
\hline Arquivos Brasileiros de Psicologia & 3 & A2/B1 \\
Psicologia: Teoria e Prática & 2 & A2/B1 \\
Avaliação Psicológica & 2 & B1/B4 \\
Revista Brasileira de Psicotera- & 2 & B3/SQ \\
pia & & \\
\hline TOTAL & 63 & \\
\hline
\end{tabular}

SQ = Sem Qualificação Qualis/Capes na área de Enfermagem

No Quadro 2 estão os informes sobre periódicos das diferentes especialidades médicas, particularmente de psiquiatria. Foram encontrados 55 artigos publicados em 12 periódicos. Quanto à qualificaçáo Capes, dos 12 periódicos 1 ( 8,33\%) não tinha qualificação tanto na medicina quanto na enfermagem - Revista da Associação Médica Brasileira -; $2(16,66 \%)$ eram B2; 4 (33,33\%) eram B3; $4(33,33 \%)$ eram B4 e $1(8,33 \%)$ era B5.

Quadro 2 - Denominação dos Periódicos de Psiquiatria/ áreas de especialidades médica; número de artigos sobre TDAH e Classificação Qualis Capes na área de Medicina e Enfermagem.

\begin{tabular}{|l|c|c|}
\hline \multicolumn{1}{|c|}{ Periódico } & $\begin{array}{c}\text { Número } \\
\text { artigos }\end{array}$ & $\begin{array}{c}\text { Qualis } \\
\text { Medicina/ } \\
\text { Enferma- } \\
\text { gem }\end{array}$ \\
\hline Jornal Brasileiro de Psiquiatria & 12 & $\mathrm{~B} 3 / \mathrm{B} 1$ \\
Arquivos de Neuro-Psiquiatria & 12 & $\mathrm{~B} 2 / \mathrm{B} 1$ \\
\hline Revista de Psiquiatria Clínica & 9 & $\mathrm{~B} 3 / \mathrm{B} 1$ \\
\hline Pediatria Moderna & 5 & $\mathrm{~B} 5 / \mathrm{B} 3$ \\
Revista Brasileira de Psiquiatria & 4 & $\mathrm{~B} 2 / \mathrm{B} 1$ \\
\hline Revista Brasileira de Neurologia & 3 & $\mathrm{~B} 4 / \mathrm{SQ}$ \\
\hline Revista Paulista de Pediatria & 3 & $\mathrm{~B} 3 / \mathrm{B} 1$ \\
\hline Jornal de Pediatria & 2 & $\mathrm{~B} 3 / \mathrm{B} 1$ \\
\hline Revista da Associação Médica & 2 & $\mathrm{SQ} / \mathrm{SQ}$ \\
Brasileira & 1 & $\mathrm{~B} 4 / \mathrm{B} 3$ \\
\hline Brasília médica & 1 & $\mathrm{~B} 4 / \mathrm{B} 3$ \\
\hline Revista de Ciências Médicas & 1 & $\mathrm{~B} 4 / \mathrm{B} 3$ \\
\hline Revista Médica de Minas Gerais & 55 & \\
\hline TOTAL & & \\
\hline
\end{tabular}

No Quadro 3 estão os periódicos com linha temática de Saúde Coletiva ou outras áreas além da Psicologia ou especialidades médicas. Foram encontrados 30 artigos publicados em 11 periódicos. Sobre a qualificação Capes - nas áreas de Educação ou Saúde Coletiva, 3 (27,27\%) eram B1, 2 (18,18\%) eram A2, $2(18,18 \%)$ eram B3, $2(18,18 \%)$ eram B4, $1(9,09 \%)$ era B5 e 1 (9,09\%) era C. Quanto ao 
Qualis Capes dos mesmos periódicos na área de Enfermagem, verificou-se: 4 (36,36\%) sem qualificação; 3 (27,27\%) eram B1; 1 (9,09\%) era A2; 1 (9,09\%) era B2; $1(9,09 \%)$ era B3 e $1(9,09 \%)$ era B5.

Quadro 3 - Denominação dos Periódicos de Educação/Saúde Coletiva / outras áreas da saúde; número de artigos sobre TDAH e Classificação Qualis Capes na área de Educação ou Saúde Coletiva e Enfermagem.

\begin{tabular}{|l|c|c|}
\hline \multicolumn{1}{|c|}{ Periódico/abreviatura } & $\begin{array}{c}\text { Número } \\
\text { artigos }\end{array}$ & $\begin{array}{c}\text { Qualis } \\
\text { Educação } \\
\text { ou Saúde } \\
\text { coletiva/ } \\
\text { Enferma- } \\
\text { gem }\end{array}$ \\
\hline $\begin{array}{l}\text { Psicopedagogia } \\
\text { Rev. bras. crescimento desen- } \\
\text { volv. hum. }\end{array}$ & 7 & $\begin{array}{c}\mathrm{B} 4 / \mathrm{B} 5 \\
\mathrm{~B} 3 / \mathrm{B} 3\end{array}$ \\
\hline Revista de Saúde Pública & 3 & $\mathrm{~A} 2 / \mathrm{A} 2$ \\
\hline Revista Ciência e Saúde Cole- \\
tiva & 2 & $\mathrm{~B} 1 / \mathrm{B} 1$ \\
Audiol. Commun & 2 & $\mathrm{~B} 1 / \mathrm{SQ}$ \\
Revista CEFAC & 1 & $\mathrm{~B} 5 / \mathrm{B} 2$ \\
\hline Rev Nufen Sér. Diálogos Diver- & 1 & $\mathrm{~B} 4 / \mathrm{SQ}$ \\
sos. & 1 & $\mathrm{~B} 3 / \mathrm{SQ}$ \\
\hline J. Soc. Bras. Fonoaudiol & 1 & $\mathrm{~B} 1 / \mathrm{B} 1$ \\
\hline Interface & 1 & $\mathrm{C} / \mathrm{SQ}$ \\
\hline Caderno de pesquias & 1 & $\mathrm{~A} 2 / \mathrm{B} 1$ \\
\hline Revista Brasileira de Educação & & \\
\hline Especial & & \\
\hline TOTAL & & \\
\hline
\end{tabular}

\section{Conclusão}

Os dados obtidos neste estudo bibliográfico ressaltam diferentes enfoques do Transtorno de Déficit de Atenção em crianças, assim como evidenciou os periódicos de maior qualificaçáo e delimitou que as áreas de psicologia e de psiquiatria são as que mais têm investigado essa temática. Os resultados deste estudo podem favorecer a orientação do profissional de saúde quanto às formas de atuaçáo neste contexto.

\section{Referências}

1. Carreiro LRR, Jorge M, Tebar MR, Moraes PF, Araujo RR, Oliveira TAER, Panhoni VACS. Importância da interdisciplinaridade para avaliaçáo e acompanhamento do transtorno do déficit de atençáo e hiperatividade. Psicol Teor Prat 2008;10(2):61-7.

2. Souza I, Mattos P, Pina C, Fortes D. TDAH: o impacto da ausência do diagnóstico. J Bras Psiquiatr 2008;57(2):139-41.
3. Pastura G, Mattos P, Araújo APQC. Prevalência do transtorno do déficit de atençáo e hiperatividade e suas comorbidades em uma amostra de escolares. Arq Neuropsiquiatr 2007;65(4a):1078-83.

4. Legnani V, Almeida SFC. A construção diagnóstica de Transtorno de Déficit de Atenção/Hiperatividade: uma discussão crítica. Arq Bras Psicol 2008;60(1):2-13.

5. Segenreich D, Fortes D, Coutinho G, Pastura G, Mattos P. Anxiety and depression in parents of a Brazilian non-clinical sample of attention-deficit/hyperactivity disorder parents of a (ADHD) students. Braz J Med Biol Res 2009;42(5):465-9.

6. Coutinho G, Mattos P, Malloy-Diniz LF. Diferenças neuropsicológicas entre crianças e adolescentes portadores de transtorno da falta de atençáo com hiperatividade e controles encaminhados por comprometimento acadêmico. Rev Bras Psiquiatr 2009;31(2):141-4.

7. Legnani VN, Almeida SFC. Hiperatividade: o 'não-decidido' da estrutura ou o 'infantil' ainda no tempo da infância. Estilos Clin 2009;14(26):14-35.

8. Santos LF, Vasconcelos LA. Transtorno do déficit de atenção e hiperatividade em crianças: uma revisão interdisciplinar. Psicol Teor Pesqui 2010;26(4):717-24.

9. Mazzoni HMO, Tabaquim ML. Distúbio de conduta e transtorno de défict de atenção/hiperatividade: uma análise diferencial. Rev Psicol 2010;1(1):63-74.

10. Andrade AC, Flores-Mendoza C. Transtorno do Déficit de Atenção/Hiperatividade: o que nos informa a investigaçấo dimensional? Estud Psicol (Natal) 2010;15(1):17-24.

11. Ferreira PVC. Uma revisão teórica sobre o transtorno de déficit de atençáo e hiperatividade (TDAH) e estratégias educacionais de atendimento ao aluno com TDAH. Rev Psicol 2011;2(2): 57.

12. França MTB. Transtorno de déficit de atenção e hiperatividade (TDAH): ampliando o entendimento. J Psicanal 2012;45(82):191-207.

13. Miranda MC. Processamento sensorial na criança com TDAH: uma revisão da literatura. Psicopedagogia 2012;29(89):256-68.

14. Costa AC, Dornelles BV, Rohde LAP. Identificação dos procedimentos de contagem e dos processos de memória em crianças com TDAH. Psicol Reflex Crit 2012;25(4):791-801.

15. Itaborahy C, Ortega F. O metifenidato no Brasil: uma década de publicaçóes. Ciênc Saude Coletiva 2013;18(3):803-16.

16. Ministério da Educação. Coordenação de Aperfeiçoamento de Pessoal de Nível Superior - CAPES [online]. [citado 2009 Abr 26]. Disponível em: URL: http:// www.capes.gov.br/.

17. Scielo Brasil. [citado 2004 Out 21]. Disponível em: URL: http: www.scielo.br

18. United States. National Library of Medicine. National Institutes of Health. Fact Sheets. What's the difference between MEDLINE and PubMed? [citado 2004 Out 25]. Disponível em URL: http://www.nlm.nih.gov/ pubs/factsheets/pubmed.html

19. Elsevier. Embase.com. [citado 2004 Out 21]. Disponível em URL: <http://www.elsevier.com.br/bibliotecadigital/embase.htm>

20. BIREME. Biblioteca Virtual em Saúde. Metodologia Lilacs. [citado 2004 out 21]. Disponível em URL: www.bireme.br/abd 